\title{
Lithium is more effective than placebo for preventing all relapses in patients with bipolar but not unipolar disorder
}

Burgess S, Geddes J, Hawton K, et al. Lithium for maintenance treatment of mood disorders. Cochrane Database Syst Rev 2001;(2):CD003013 (latest version Sep 27 2000).

\section{QUESTION: In patients with recurrent mood disorder, is lithium more effective than placebo for preventing relapse?}

Source of funding: South East region NHSE Research and Development UK.

For correspondence: $\mathrm{Dr}$ $J$ Geddes, Department of Psychiatry, University of Oxford, Warneford Hospital, Oxford OX3 $7 / X, U K$ Fax $+44(0) 1865793$ 101.

\section{Data sources}

Published and unpublished randomised controlled trials were identified by searching the Cochrane Controlled Clinical Trials Registers; by reviewing the reference lists of all identified trials, other relevant papers, and major textbooks on mood disorder; by handsearching the journals Lithium (1990-4) and Lithium Therapy Monographs (1987-91); and through personal contact with authors of trials included in the review, with other recognised experts, and with pharmaceutical companies.

\section{Study selection}

Studies were selected if patients were diagnosed with mood disorder (primarily bipolar disorder and recurrent unipolar disorder), lithium was compared with placebo as maintenance treatment, and follow up was $\geqslant 3$ months. Trials that had lithium combined with another treatment, had unsound diagnoses, were categorised as

Lithium v placebo in patients with bipolar or unipolar disorder, followed for a period of 11 months to 4 years*

\begin{tabular}{llllll} 
Disorder & Relapse & \multicolumn{2}{l}{ Weighted event rates } & RRR (95\% Cl) & NNT (CI) \\
\hline \multirow{2}{*}{ Bipolar } & Lilthium & Placebo & \\
\hline & Manic & $33 \%$ & $61 \%$ & $40 \%(13$ to 59$)$ & 4 (2 to 30$)$ \\
\hline \multirow{2}{*}{ Unipolar } & Depressive & $8.7 \%$ & $20 \%$ & $44 \%(-9$ to 72$)$ & Not significant \\
\hline & Manic & $21 \%$ & $63 \%$ & $50 \%(0$ to 75$)$ & Not significant \\
\hline & Depressive & $16 \%$ & $56 \%$ & $52 \%(-7$ to 79$)$ & Not significant \\
\hline
\end{tabular}

*Abbreviations defined in glossary; RRR, NNT, and Cl calculated from data in article. All meta-analyses were done with a random effects model. awaiting assessment, or were discontinuation studies were excluded.

\section{Data extraction}

2 reviewers independently extracted data on patient characteristics, intervention details, and outcome measures. 2 different reviewers independently assessed study quality using the Cochrane Criteria for quality assessment and the Jadad scale. Main outcome was relapse (as defined by the authors).

\section{Main results}

Of 9 studies $(\mathrm{n}=825)$ that met the inclusion criteria, 3 studies $(n=412)$ were in patients with bipolar disorder and 3 studies $(n=196)$ were in patients with unipolar disorder. This abstract does not include results from 4 studies with mixed diagnoses of mood disorder. Patients were followed until relapse or for a period of 11 months to 4 years. Lithium concentrations were reported in 8 of the 9 studies and ranged from 0.5 to $1.4 \mathrm{mmol} / \mathrm{l}$. In patients with bipolar disorder, lithium was more effective than placebo for preventing all relapses (table). Lithium was not more effective than placebo for preventing all relapses in patients with unipolar disorder, or for preventing manic and depressive relapses in patients with unipolar or bipolar disorder (table).

\section{Conclusions}

Lithium is more effective than placebo for preventing all relapses in patients with bipolar disorder. Lithium is not more effective than placebo for preventing all relapses in patients with unipolar disorder, or for preventing manic and depressive relapses in patients with bipolar or unipolar disorder.

\section{COMMENTARY}

This well designed review by Burgess et al addresses an important clinical question, given the recurrent nature of mood disorders. The question they ask is controversial because results showing fewer relapses in patients on lithium maintenance treatment have been criticised for bias from the effect of discontinuation. ${ }^{1}$ In discontinuation studies, patients who were stable on lithium for a period of time were randomised to continued lithium or placebo, often leading to an abrupt discontinuation of lithium in the placebo group. Such discontinuation has been associated with a higher rate of relapse in those stopping lithium, ${ }^{2}$ resulting in misleading differences between the 2 groups. Methodologically, one of the strengths of this review is that discontinuation studies were specifically excluded, though discontinuation of lithium in a proportion of patients in most of the remaining studies could not be excluded. This approach necessarily limits the number of studies, and hence the number of patients, included in the review, and the precision of its estimates. The conclusions of the review should be considered with these points in mind.

This review finds that evidence exists to suggest that lithium maintenance treatment reduces the overall risk of relapse in bipolar affective disorder, with 1 relapse being prevented for every 4 patients taking lithium. Whether this effect was driven more by reductions in depressive or manic relapse, or both, is statistically uncertain; the overall relapse prevention is the headline. The place of lithium maintenance treatment in unipolar affective disorder remains unclear; there were only 3 studies to include in the review. Lithium has not become established as a routine prophylactic agent in unipolar disorder. The recommendation is to do more trials.

The authors also highlight the lack of data on health and social functioning, patients' attitudes to treatment, and mortality and suicide in lithium maintenance studies to date. This review helps confirm the usefulness of lithium for treatment of bipolar affective disorder.

Noel Kennedy, MB, MSc, MRCPsych Peter B Jones, PhD, MB, MRCP, MRCPsych Cambridge University Cambridge, UK

1 Moncrieff J. Lithium: evidence reconsidered. Br J Psychiatry 1997;171:113-9.

2 Goodwin GM. Recurrence of mania after lithium withdrawal. Implications for the use of lithium in the treatment of bipolar affective disorder, Br $J$ Psychiatry 1994;164:149-52. 